\title{
Registro del primer caso de albinismo completo en Desmodus rotundus en Argentina
}

\author{
Ramirez, N.N.; Bottinelli, O.R.; Ruiz, R.M.; Bastos, R.S. \\ Servicio de Extensión Salud Pública Veterinaria, Cátedra de Salud Pública, Facultad de Ciencias Veterinarias \\ UNNE, Sargento Cabral 2139, Corrientes (3400), Argentina. Tel: 03783-425753. \\ E-mail: natyramirez13@hotmail.com.
}

\begin{abstract}
Resumen
Ramirez, N.N.; Bottinelli, O.R.; Ruiz, R.M.; Bastos, R.S.: Registro del primer caso de albinismo completo en Desmodus rotundus en Argentina. Rev. vet. 21: 1, 63-65, 2010. El albinismo puede ser heredado por reproducción directa de individuos albinos o bien indirectamente debido al cruzamiento de individuos recesivos con coloración normal. El albinismo verdadero consiste en la ausencia total de pigmentación, pero existen otras formas de despigmentación, consideradas como albinismo parcial. Los casos de albinismo completo son muy raros, particularmente en animales silvestres y muy especialmente en murciélagos, sin embargo se hallaron casos en veintidós países a nivel mundial. En Sudamérica existen trece casos registrados, uno de ellos en Argentina, en un murciélago de la Provincia de Salta. En este reporte se comunica un nuevo hallazgo acaecido en el Departamento Bella Vista, Provincia de Corrientes (Argentina), que constituye el primer caso de albinismo completo registrado en este país en la especie Desmodus rotundus. Estos descubrimientos son de relevancia debido a que es necesario el registro de casos de albinismo en diferentes especies, no solo por fines identificatorios sino también por estudios genéticos, etológicos y ecológicos, así como sus implicancias en la distribución de enfermedades.
\end{abstract}

Palabras clave: Desmodus rotundus, albinismo, Corrientes, Argentina.

\begin{abstract}
Ramirez, N.N.; Bottinelli, O.R.; Ruiz, R.M.; Bastos, R.S.: First report of complete albinism in Desmodus rotundus species from Argentina. Rev. vet. 21: 1, 63-65, 2010. Albinism can be inherited by direct reproduction of albino specimens or indirectly by crossing of recessive individuals of normal color. True albinism is the total absence of pigmentation, but there are other forms of depigmentation considered as partial albinism. Cases of complete albinism are exceptional, in particular in wild animals and especially in bats, but cases were reported already in twenty-two countries worldwide. In South America there are thirteen recorded cases, one of them in a bat from Salta, north-western Argentina. In this article we report a new case of albinism in a bat found in Corrientes, north-eastern Argentina, being at the same time the first for the Desmodus rotundus species. Reporting of albinism cases are of great importance not only for taxonomic description purposes but also for genetic, ethological and ecological studies, and in particular for this species regarding its role in the spread of some diseases.
\end{abstract}

Key words: Desmodus rotundus, albinism, Corrientes, Argentina.

\section{INTRODUCCIÓN}

El albinismo verdadero consiste en la ausencia total de pigmentación y se caracteriza por la carencia de pigmento en piel, pelaje e iris de los ojos ${ }^{17}$. Existen otras formas consideradas como albinismo parcial o leucismo, donde la piel posee en cierta manera pigmentación normal, pelo o escamas blancas y pigmentación oscura en ojos y uñas ${ }^{9}$. Los casos de albinismo completo no son comunes, particularmente en animales silvestres ${ }^{17}$

Recibido: 22 abril 2010 / Aceptado: 11 mayo 2010
, no obstante en murciélagos fueron registrados en 22 países ${ }^{2}$. Hasta el año 2004 se reconocieron 67 casos de albinismo verdadero en 40 especies diferentes de murciélagos en todo el mundo ${ }^{16}$, de las cuales seis han sido registradas para Sudamérica ${ }^{14}$.

Nuestras últimas actualizaciones bibliográficas corroboran un aumento en el número de casos de albinismo verdadero en quirópteros, como muestra la Tabla 1. En Sudamérica existen 13 casos registrados, uno de ellos en Argentina, Provincia de Salta, en el año 2003 ${ }^{4}$. El último caso registrado corresponde a Brasil, año 2008, para la especie Artibeus cinereus ${ }^{11}$. El fenómeno 
de albinismo es relevante porque, como cualquier condición variable, puede proveer evidencia indirecta de variabilidad genética subyacente, como está citado en otras especies ${ }^{6}$.

El hallazgo se produjo durante el desarrollo de actividades inherentes a un proyecto de vigilancia ecoepidemiologica que aspira a solucionar la problemática del vampiro Desmodus rotundus en la producción ganadera, por su responsabilidad en la circulación del virus de la rabia paresiante y el consecuente riesgo para la salud humana. El principal objetivo del trabajo es reducir la densidad poblacional de la principal especie que opera como reservorio de la rabia paresiante por debajo del umbral necesario para que la enfermedad se mantenga en esa población.

\section{MATERIAL Y MÉTODOS}

La captura se llevó a cabo en una colonia de Desmodus rotundus descubierta en el Paraje Isla Alta, $4^{\circ}$ Sección del Departamento de Bella Vista, Provincia de Corrientes (Argentina) el 12 de septiembre de 2008. Para la captura se utilizó una red de mano. El objetivo perseguido era realizar estudios de identificación de especies, morfometría y localización geográfica de las colonias. La clave utilizada para la identificación de la especie se basó en una guía originaria de la Universidad de Oklahoma ${ }^{3}$.

\section{RESULTADOS Y DISCUSIÓN}

Como indica la Figura 1, fue identificado un ejemplar de Desmodus rotundus con ojos de color rojo y despigmentación total en ventral y dorsal del cuerpo y alas, características que ratificaron la existencia de albinismo completo. En Sudamérica fueron registrados

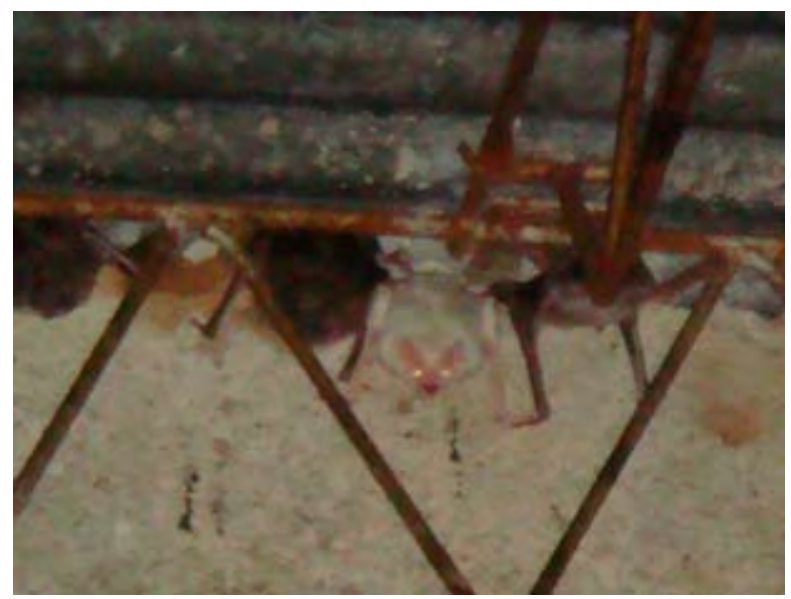

Figura 1. Ejemplar de Desmodus rotundus con albinismo verdadero. seis casos de albinismo completo de la especie Desmodus rotundus ${ }^{18}$, con el presente hallazgo sumarían siete los casos y el primero en Argentina para esta especie. El descubrimiento asume importancia porque las variaciones genéticas pueden estar influenciadas por factores ecológicamente relevantes. Muchos estudios de sobrevivencia ${ }^{12}$, de comportamiento ${ }^{13} \mathrm{y}$ de asociación con diferentes patologías ${ }^{5,7}$, son realizados en ejemplares albinos de especies como venados ${ }^{13} \mathrm{y}$ el propio Desmodus rotundus ${ }^{18}$.

El albinismo ha sido descripto en reptiles ${ }^{8}$, aves ${ }^{10}$, lobos marinos ${ }^{1}$ y peces ${ }^{15}$. El registro de casos de albinismo de diferentes especies animales a nivel mundial, es necesario no solo por la identificación, sino también por sus relaciones con el comportamiento animal y la genética, así como implicancias con la ecología y distribución de enfermedades como la rabia paresiante en el caso de los murciélagos hematófagos.

Agradecimientos. A Federico Ledesma y Francisco Iriondo por su ayuda en las tareas de campo, así como al veterinario Lino Castillo por su colaboración en la localización de la colonia de murciélagos.

\section{REFERENCIAS}

1. Acevedo J, Aguayo M. 2008. Leucistic South American sea lion in Chile, with a review of anomalously color in otariids. Rev Biol Marina \& Oceanogr 43: 413-417.

2. Aul B, Marimuthu BA. 2006. Sighting of an albino bat in a colony of cave-dwelling microchiropteran, Hipposideros diadema nicobarensis at the Nicobar Islands. Current Sci 90: 912-914.

3. Barquez RM, Giannini NP, Mares MA. 1993. Guide to the bats of Argentina. Publ. Oklahoma Museum of Natural History, Univ. of Oklahoma, Norman, 119 p.

4. Barquez RM, Carrrizo LV, Ferro LI, Flores DA, Mollerach MI, Sánchez MS, García López AP. 2003. Primeiro caso de albinismo total para Sturnira erythromos (1844) (Chiroptera: Phyllostomidae). Chiroptera Neotrop 9: 166-169.

5. Brismar J, Harfi, HA. 1992. Partial albinism with immunodeficiency: a rare syndrome with prominent posterior fossa white matter changes. Amer J Neuroradiol 13: 387393. 
6. Jehl JR. 1985. Leucism in pared grebes in western North America. The Condor 87: 439-441.

7. Jeffery G, Williams A. 1994. Is abnormal retinal development in albinism only a mammalian problem? Normality of a hypopigmented avian retina. Exp Brain Res 100: 47-57.

8. Martínez-Silvestre A, Soler J, Gener JM, García M, Martí C. 2009. Albinismo total de Coronella girondica en la Península Ibérica. Bol Asoc Herpetol Esp 20: 44-45.

9. Miller JD. 2005. All about albinism. Missouri Conservationist 66: 5-7.

10. Morici A. 2009. Leucismo en loica común (Sturnella loyca) en el sudoeste de la Provincia de Buenos Aires, Argentina. Nuestras Aves 54: 8.

11. Oliveira HF, Aguiar LM. 2008. A new case of complete albinism in a bat from Brasil. Chiroptera Neotrop 14: 421-423.

12. Parsons GJ, Bonderup-Nielsen S. 1995. Partial albinism in an island population of Meadon Voles, Microtus pennsylvanicyus, from Nova Scotia, Canadian Field Naturalist 109: 263-264.
13. Rodrigues FH, Silveira L, Jácomo AT, Emygdio LA, Monteiro F. 1999. Um albino parcial de veado campeiro (Ozotoceros bezoarticus, Linnaeus) no Parque Nacional das Emas, Goiás. Rev Bras Zool 16: 1229-1232.

14. Roncancio N, Ramirez-Chaves HE. 2008. Registro de leucismo en Sturnira erythromos en los Andes centrales de Colombia. Chiroptera Neotrop 14: 412-414.

15. Sazima I, Pombal J. 1986. Um albino de Rhamdella minuta com notas sobre comportamiento (Osteichthyes, Pimelodinae). Rev Bras Biol 46: 377-381.

16. Sodré MN, Uieda W, Baldim M. 2004. First record of albinism in the bat Eumops glacinus (Molossidae) from Southeastern Brazil. Chiroptera Neotrop 10: 200-201.

17. Uieda W. 2000. A review of complete albinism in bats with five new cases from Brazil. Acta Chiropterologica 2: 97 105.

18. Uieda W. 2001. Behavior of an albino vampire bat, Desmodus rotundus (E. Geoffroy) (Chiroptera, Phyllostomidae) in captivity. Rev Bras Zool 18: 641-644. 